\title{
AN IMPROVED LIMIT FOR THE RATE OF PERIOD CHANGE IN THE ZZ CETI STAR G117-B15A \\ or \\ The Most Stable Optical Clock Known
}

\author{
S.O.Kepler \\ Instituto de Fisica \\ Universidade Federal do Rio Grande do Sul \\ Brazil \\ and \\ D.E.Winget, E.L.Robinson, and R.E.Nather \\ McDonald Observatory and Department of Astronomy \\ The University of Texas at Austin \\ U.S.A.
}

\begin{abstract}
The rate of change of period with time for the g-mode pulsations in ZZ Ceti stars depends directly on the cooling timescale for a DA white dwarf, and therefore a measurement of its value can yield an evolutionary timescale for the white dwarf. We have obtained an upper limit for the rate of change of the period of the dominant pulsation in the light curve of the ZZ Ceti star G117-B15A of $|d P / d t| \leq 9.9 \times 10^{-16} \mathrm{8} / \mathrm{s}$ at the $68 \%$ confidence level, equivalent to a timescale for period change of $|P / \dot{P}| \geq 6.9 \times 10^{8} \mathrm{yr}$.
\end{abstract}

\section{INTRODUCTION}

The ZZ Ceti stars are the single, normal DA white dwarfs that show luminosity variations. Fontaine et. al. (1982) and Greenstein (1982), using a homogeneous set of colors, found that all observed DA white dwarfs in the color range corresponding to effective temperatures in a narrow strip ( $1400 \mathrm{~K}$ wide) around $11000 \mathrm{~K}$ were $\mathrm{ZZ}$ Ceti stars. There are 19 known ZZ Cetis, all multiperiodic, with periods ranging from 109 to $1186 \mathrm{~s}$ and peak-to-peak light variations up to 34\%. The observed variations are due to nonradial g-mode pulsations (Kepler 1984) and, since each periodicity constrains independently the properties and structure of the star, they can ideally be used to infer the whole structure of the white dwarf, as demonstrated by the mode trapping calculations of Winget et. al. (1982). For a review of the properties of the ZZ Ceti stars, see the article by Don Winget in these Proceedings.

The timescale for period change for the pulsations observed in the ZZ Ceti stars is directly proportional to the cooling timescale of the white dwarfs, and this timescale can be transformed to the white dwarf birth rate through the measurement of their space densities. The rate of period change can also give directly the core composition, since the cooling timescale is directly related to the equation of state of the white dwarf core and can, in principle, be used to distinguish among white dwarfs of different core compositions. (Robinson and Kepler 1980). The rate of period change is, therefore, and important quantity to measure. We report on our continuing observations of one of the ZZ Ceti stars, G117-B15A, in which we hope to measure a change in pulsation period.

\section{OBSERVATIONS}

In 1982 we used our 85 hours of high speed photometry on the star to show that the light curve of G117-B15A has at least six pulsation modes simultaneously excited with periods of $107.6 \mathrm{~s}$, $119.8 \mathrm{~s}, 126.2 \mathrm{~s}, 215.2 \mathrm{~s}, 271.0 \mathrm{~s}$, and $304.4 \mathrm{~s}$. The $215.2 \mathrm{~s}$ pulsation has a fractional semiamplitude of 0.022 , nearly 3 times greater than any of the other pulsations, and dominates the light curve 
completely. We could then derive an upper limit for the rate of change of period with time of $|\dot{P}| \leq 7.8 \times 10^{-14} \mathrm{~s} / \mathrm{s}$ for this pulsation (Kepler, Robinson, Nather and McGraw 1982). Using additional data, we were later able to improve the limit to $|\dot{P}| \leq 2.0 \times 10^{-14} \mathrm{~s} / \mathrm{s}$ (Kepler, Robinson and Nather 1982).

We have obtained an additional 53 hours of high speed photometry using a two-star photometer (Nather 1973) on the $2.1 \mathrm{~m}$ Struve telescope at McDonald Observatory to improve our estimate of the rate of period change for this pulsation. The data now span 10.8 years. The observations were obtained with a blue-sensitive bialkali phototube (an RCA 8850) and, as G117-B15A is rather faint, $V=15.52$ (Eggen and Greenstein 1965), all measurements were obtained in unfiltered light to improve the photon detection rate. The amplitudes observed should be used with caution, since they are wavelength dependent (Robinson, Kepler, and Nather 1982), and the effective wavelength of our white-light photometry is not well determined. Table 1 gives a journal of the observations. The method for data reduction and conversion of the time base from UTC to the more uniform Barycentric Julian Ephemeris Date was described in Kepler et. al. (1982).

Table 1

\begin{tabular}{cccccr}
\hline Run & $\begin{array}{c}\text { Length of } \\
\text { Observation } \\
(\mathrm{hr})\end{array}$ & $\begin{array}{c}\text { Integration } \\
\text { Time }(\mathrm{sec})\end{array}$ & $\begin{array}{c}\text { Fractional } \\
\text { Semi- } \\
\text { Amplitude }\end{array}$ & $\begin{array}{c}\text { BJED at Maximum } \\
(\mathbf{2 4 4} 0000.0+)\end{array}$ & $\begin{array}{r}\text { Error } \\
(\mathrm{sec})\end{array}$ \\
\hline 2593 & 0.9 & 10 & 0.024 & 4637.776174 & 1.6 \\
2597 & 7.0 & 10 & 0.023 & 4641.624287 & 0.5 \\
2629 & 2.1 & 10 & 0.022 & 4992.789531 & 1.2 \\
2633 & 6.6 & 10 & 0.021 & 4994.689956 & 0.7 \\
2637 & 3.4 & 10 & 0.024 & 4996.744801 & 0.8 \\
2640 & 3.7 & 10 & 0.024 & 4997.723649 & 0.7 \\
JES1 & 2.9 & 10 & 0.021 & 5021.716661 & 1.0 \\
2869 & 3.2 & 10 & 0.022 & 5703.860004 & 1.4 \\
2870 & 5.6 & 10 & 0.028 & 5734.642701 & 0.7 \\
2872 & 2.4 & 10 & 0.025 & 5735.643972 & 0.8 \\
ER60 & 3.8 & 5 & 0.023 & 6113.763716 & 0.7 \\
ER110 & 1.8 & 5 & 0.021 & 6443.775386 & 1.0 \\
3096 & 5.9 & 5 & 0.023 & 6468.630178 & 0.5 \\
3106 & 1.2 & 10 & 0.024 & 6473.718679 & 0.9 \\
3141 & 1.0 & 10 & 0.022 & 6523.620086 & 1.3 \\
3143 & 1.0 & 10 & 0.020 & 6524.613917 & 1.3 \\
\hline
\end{tabular}

3. THE UPPER LIMIT TO $\dot{P}$

Our method of measuring the rate of period change is as follows: first, we fit a sinusoid with a period of $215.2 \mathrm{~s}$ to the data by least squares to obtain the mean time of arrival for the maximum of the pulsation for each night's data. After converting the observed times of maxima to times that would be observed at the barycenter of the solar system, we calculate the difference between the Observed times of maxima and the times of maxima Calculated using a linear ephemeris, obtaining therefore an (O-C) diagram. Unique cycle counts $E$ were obtained for each night since the period derived in 1982 was extremely accurate, permitting us to bridge the whole data base without any possibility of cycle count error. The fairly large apparent scatter in the diagram is an artifact of the varying quality of the different runs; no data point lies more than $3 \sigma$ away from the mean.

The rate of period change for the pulsation, as well as a correction to the period and phase, can then be obtained by fitting a parabola to the (O-C) diagram.

Fitting a parabola by weighted least-squares to the 52 times of maxima obtained since 1975, one for each night of data, we obtain:

$$
E_{0}=\text { BJED 2442397.917508 } \pm 0.000008 \text { days }
$$




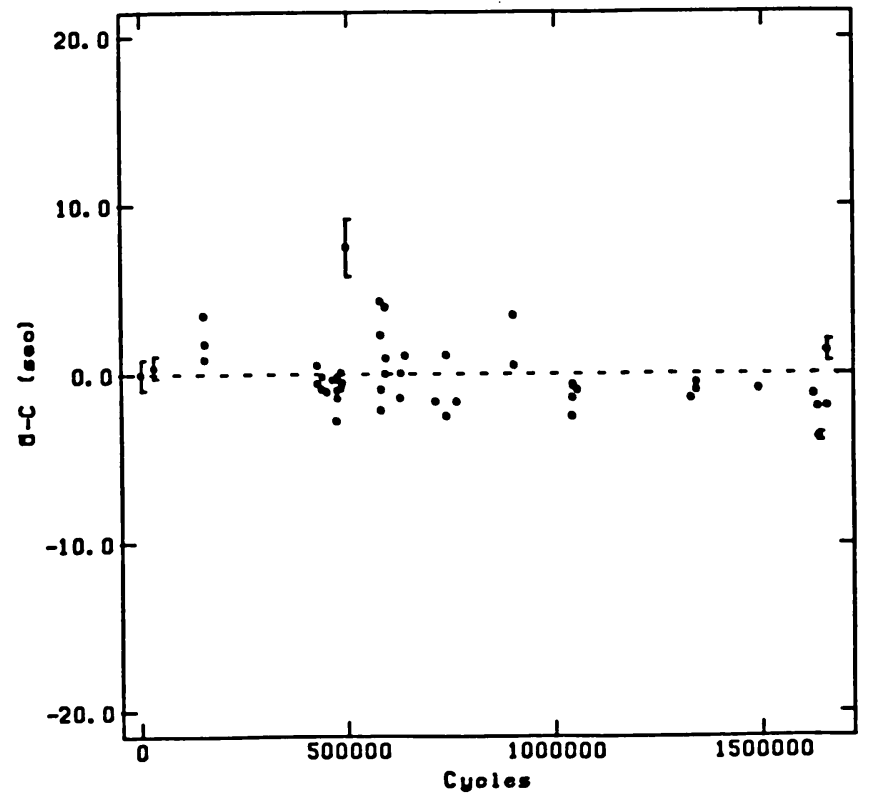

Figure 1. The (O-C) diagram for the $215.2 \mathrm{~s}$ pulsation of G117-B15A. The observed times of maxima are listed in Table 1 , and the calculated times of maxima were derived using a linear ephemeris starting at BJED 2442397.917508.

$$
\begin{aligned}
& P=215.1973905 \pm 0.0000017 \mathrm{sec} \\
& d P / d t=-(5.7 \pm 7.8) \times 10^{-15} \mathrm{~s} / \mathrm{s}
\end{aligned}
$$

which is equivalent to an upper limit for the rate of period change of

$$
|d P / d t| \leq 9.9 \times 10^{-15} \mathrm{~s} / \mathrm{s}
$$

at the $68 \%$ confidence level, or

$$
|P / \dot{P}| \geq 6.9 \times 10^{8} \mathrm{yr} .
$$

In other words, there is no detectable change in the period of the $215.2 \mathrm{~s}$ pulsation of G117-B15A.

\section{DISCUSSION}

The observational upper limit on the rate of period change can be compared with the expected theoretical rate of period change of nonradial g-mode pulsations calculated by Winget (1981), caused by the cooling of the white dwarf. His calculations were made with a linear, nonadiabatic pulsation code, and used evolutionary models for carbon core white dwarfs incorporating stratified $\mathrm{H} / \mathrm{He}$ envelopes consistent with diffusion equilibrium. For $0.6 M_{\odot}$ models corresponding to the ZZ Ceti stars, he found $P / \dot{P} \approx 4 \times 10^{9}$ yr for a pure carbon core white dwarf and modes with low $k$ and $\ell$ values. Since the timescale for period change is inversely proportional to the mean atomic weight of the core composition, the timescale for a white dwarf with Fe core can be as short as $P / P \approx 7.3 \times 10^{8} \mathrm{yr}$, for a $\ell=1$ and $k=1 \mathrm{~g}$-mode. Our results are therefore still consistent with the theoretical expectations for $g$-modes. Since our limit on $P / \dot{P}$ will continue to improve quadratically with the time baseline as the star is observed, we expect to obtain a measurement accuracy comparable with the predicted time scale in a few years. 
In order to measure a rate of change of period with time of the order of $10^{-14} \mathrm{~s} / \mathrm{s}$, like the one observed in G117-B15A, there is a very stringent restriction on the dynamical environment of the white dwarf, since even a planet of the mass of Jupiter, and at the same distance from the white dwarf that Jupiter is from the Sun, would give an apparent rate of change of the pulsation period, i.e., of the arrival time of the pulses of the order of $10^{-12} \mathrm{~s} / \mathrm{s}$. That means that to give a $\dot{P}$ of the order of $10^{-14} \mathrm{~s} / \mathrm{s}$, the white dwarf cannot have any body of even planetary mass orbiting it. The technique of measuring rates of change of periods with time may therefore prove itself ultimately capable of detecting planets outside the solar system, in addition to producing information on the stellar structure and evolution.

\section{REFERENCES}

Eggen, O.J., and Greenstein, J.L. 1965, Ap. J., 141, 183.

Fontaine, G., McGraw, J.T., Dearborn, D.S.P., Gustafson, J., and Lacombe, P. 1982, Ap. J., 258, 651.

Greenstein, J.L. 1982, Ap. J., 258, 661.

Kepler, S.O. 1984, Ap. J., 286, 314.

Kepler, S.O., Robinson, E.L. and Nather, R.E. 1982, in "Pulsations in Classical and Cataclysmic Variable Stars," ed. by J.P. Cox and C.J. Hansen (Boulder, CO: J.I.L.A.), p. 73.

Kepler, S.O., Robinson, E.L., Nather, R.E., and McGraw, J.T. 1982, Ap. J., 254, 676.

Nather, R.E. 1973, Vistas in Astronomy, 15, 91.

Robinson, E.L., and Kepler, S.O. 1980, Spa. Sci. Rev., $27,613$.

Robinson, E.L., Kepler, S.O., and Nather, R.E. 1982, Ap. J., 259, 219.

Winget, D.E. 1981, Ph.D. Thesis, University of Rochester.

Winget, D.E., Van Horn, H.M., Tassoul, M., Hansen, C.J., Fontaine, G., and Carrol, B.W. 1982, Ap. J., 252, L65. 